2. Borawski J, Wilczyńska-Borawska M, Stokowska W, Myśliwiec M. The periodontal status of pre-dialysis chronic kidney disease and maintenance dialysis patients. Nephrol Dial Transplant 2007; 22: 457-464

3. Trzonkowski P, Mysliwska J, Dębska-S?lizień A et al. Longterm therapy with recombinant human erythropoietin decreases percentage of $\mathrm{CD} 152^{+}$lymphocytes in primary glomerulonephritis haemodialysis patients. Nephrol Dial Transplant 2002; 17: 1070-1080

4. Tyrzyk S, Sadlak-Nowicka J, Kędzia A, Bochniak M, Szumska-Tyrzyk B, Rutkowski P. Clinical and mycological examinations of oral mucosa in cyclosporine A treated patients after renal transplantation. Przegl Lek 2004; 61: 467-472

5. Tyrzyk S, Sadlak-Nowicka J, Kędzia A, Bochniak M, Rutkowski P. Proposal of a periodontal preventive and treatment scheme for patients before and after renal transplantation receiving cyclosporine A. Dent Med Probl 2006; 43 : 483-491

6. Stellingsma C, Vissink A, Meijer HJA, Kuiper C, Raghoebar GM. Implantology and the severely resorbed edentulous mandible. Crit Rev Oral Biol Med 2004; 15: 240-248

7. Wojtowicz A, Grabowska K, Kukuła K et al. Densitometric examination of male patients with renal osteodystrophy. Prot Stom 2002; 52: 195-201

8. Kukuła K, Wojtowicz A, Dijakiewicz M et al. The linear rediometric analysis of the jaws in renal osteodystrophy: introduction to implanto-prosthodontic treatment. Prot Stom 2005; 55: 336-343

9. Wojtowicz A, Dijakiewicz M, Wandzel B et al. The evaluation of mineral crystallinity of mandibular bone tissue using electron paramagnetic resonance (EPR) in patients suffering from renal osteodystrophy. Przegl Lek 2006; 63: 759-761

10. Dijakiewicz M, Wojtowicz A. Problems and algorithm of dental procedures in patients on renal replacement therapy. In: Rutkowski B, ed. Dialysis in Medical Practice, MAKmedia, Gdańsk: 2004

doi:10.1093/ndt/gfm317

Advance Access publication 25 May 2007

\section{Calcimimetic increases osteoprotegerin and decreases fetuin-A levels in dialysis patients}

Sir,

The calcimimetic drug cinacalcet, recently introduced as therapy for Secondary Hyper-Parathyrodism (SHP) in dialysis patients, has greatly enhanced the ability to achieve simultaneous control of parathyroid hormone (PTH), calcium and phosphate [1]

However, there have been no data to show that a better control of mineral metabolic parameters is matched with improved clinical outcomes of vascular calcification processes, and hence, of vascular morbidity and mortality.

Recent studies have shown that both osteoprotegerin (OPG) and Fetuin-A are associated with the vascular calcification process in uraemic patients [2,3].

Of 164 patients from our dialysis centre, we submitted 29 of these having serum i-PTH $>300 \mathrm{pg} / \mathrm{ml}$ while on standard therapy (calcitriol + phosphate binders) to cinacalcet treatment over a 6-month period.

After an observation period of 3 weeks that included the standard therapy, cinacalcet was started at an initial dose of $30 \mathrm{mg}$ per day. Thereafter, cinacalcet, vitamin D metabolites and phosphate binder doses were adjusted according to
i-PTH, c-Ca and Pi levels. Cinacalcet was allowed to be increased by $30 \mathrm{mg}$ steps every 15 days to a maximal allowed dose of $180 \mathrm{mg}$ per day. The drug was reduced or withdrawn if i-PTH levels were $<150 \mathrm{pg} / \mathrm{ml}$, if c-Ca was $<8.4 \mathrm{mg} / \mathrm{dl}$, or if any adverse events appeared.

Blood levels of i-PTH, c-Ca, inorganic phosphate $(\mathrm{Pi})$, albumin, IL-6, Fetuin-A, OPG were assessed at baseline and at the end of the study period. Intact PTH, c-Ca and Pi levels were also checked every 15 days. Over the 6-month study period, the doses of cinacalcet, vitamin $\mathrm{D}$ metabolites, phosphate binders, erythropoietin (EPO), anti-hypertensive (antiHT) drugs and any major and minor adverse events were recorded.

OPG and Fetuin-A were assessed by ELISA (Normal values: OPG $4.1 \pm 0.33 \mathrm{pmol} / \mathrm{l}$; Fetuin-A $0.35-0.95 \mathrm{~g} / \mathrm{l})$.

IL-6 was determined with a solid-phase, enzyme-labelled, chemiluminescent sequential immunometric assay (Normal values were $0-5.9 \mathrm{pg} / \mathrm{ml}$ ).

Five of the original 29 patients did not complete the study (two due to cardio-vascular deaths; one for withdrawal after a non-fatal cardio-vascular event; two for receiving renal transplantation), resulting in 24 patients included in the final analysis (16 males; aged 38-78).

Table 1 shows the effects of cinacalcet treatment on i-PTH, c-Ca, Pi and the c-Ca $\times$ Pi product. All of these parameters were significantly decreased after cinacalcet therapy.

The mean dose of cinacalcet utilized at the end of the study was $63.12 \pm 40.3 \mathrm{mg}$ per day. There were no significant changes in Vitamin D, Sevelamer, Ca based Pi binder, antiHT drugs or EPO doses.

Following cinacalcet treatment, mean serum OPG levels were significantly increased and serum Fetuin-A levels were significantly decreased (Table 2). On the other hand, there were no significant changes in mean IL-6 values. OPG increments were significantly correlated with the degree of c-Ca reduction $(r=0.445 ; \quad P=0.029)$, whereas Fetuin-A

Table 1. Effects of 6-month Cinacalcet treatment on the main mineral metabolic parameters and doses of concomitant therapy $($ mean $\pm \mathrm{SD})$

\begin{tabular}{lccc}
\hline & Baseline & 6th month & $P$ \\
\hline i-PTH pg/ml & $714.0 \pm 410.1$ & $379.8 \pm 439.1$ & 0.0002 \\
$\mathrm{c}-\mathrm{Ca} \mathrm{mg} / \mathrm{dl}$ & $10.52 \pm 0.94$ & $9.10 \pm 0.72$ & 0.00001 \\
$\mathrm{Pi} \mathrm{mg/dl}$ & $4.93 \pm 1.33$ & $4.20 \pm 1.97$ & 0.0032 \\
$\mathrm{Ca} \times \mathrm{Pi} \mathrm{mg} / \mathrm{dl}^{2}$ & $51.9 \pm 14.9$ & $38.1 \pm 10.5$ & $<0.00001$ \\
$\begin{array}{l}\text { Vitamin D } \\
\quad \text { therapy }(\mu \mathrm{g} / \text { week) }\end{array}$ & $2.05 \pm 1.84$ & $1.63 \pm 1.36$ & 0.331 \\
$\begin{array}{l}\text { Ca carbonate } \\
\text { (g/day of }\end{array}$ & $0.442 \pm 0.754$ & $0.480 \pm 0.556$ & 0.726 \\
$\quad$ elemental Ca) & & & \\
Sevelamer (g/day) & $5.43 \pm 2.90$ & $5.00 \pm 2.80$ & 0.319 \\
\hline
\end{tabular}

Table 2. Effects of cinacalcet on OPG, Fetuin-A and IL-6 levels $($ mean $\pm \mathrm{SD})$

\begin{tabular}{lrrl}
\hline & Baseline & 6th month & \multicolumn{1}{c}{$P$} \\
\hline OPG (pmol/l) & $16.61 \pm 13.4$ & $22.20 \pm 16.7$ & 0.001 \\
Fetuin-A (g/l) & $0.766 \pm 0.44$ & $0.430 \pm 0.24$ & 0.01 \\
IL-6 (pg/ml) & $5.75 \pm 4.37$ & $5.93 \pm 5.19$ & 0.847 \\
\hline
\end{tabular}


reductions were correlated with reductions in i-PTH levels $(r=0.495 ; P=0.024)$. There were no relationships between IL-6 changes and the main mineral metabolism related parameters.

Our results agree with previous experimental findings [4] and point to the efficacy of calcimimetic drugs for the control of uraemic SHP.

It was unexpected that 6 months of cinacalcet treatment resulted in highly significant increases in OPG and decreases in Fetuin-A serum levels.

OPG, a cytokine produced and secreted mainly by osteoblasts, has been claimed to play an as yet undefined role in the vascular calcification process. Although a protective effect of increased OPG levels on vascular calcification has been suggested, higher OPG serum levels have been linked to an increased extent of arterial wall calcification, increased mortality rates in uraemic patients, and most importantly, with increased mortality in dialysis patients $[3,5]$. The clinical significance of the OPG increase observed in our patients and its potential effects on the vascular calcification process cannot be drawn from our data. Interestingly, OPG increases were significantly correlated with the degree of reduction in c-Ca levels. In association with the OPG increases, cinacalcet treatment also caused significant decreases in Fetuin-A levels without changes in IL-6, indicating no change in the inflammatory state in our patients. The Fetuin-A reduction was significantly related to PTH decreases. Previous studies have emphasized an association between low Fetuin-A levels with both increased vascular calcification and cardiovascular mortality $[2,6]$. From our data, we cannot determine whether the Fetuin-A decrease represents a real increase in risk for the calcification process, or whether it is the consequence of a reduced demand for a feedback defence mechanism, which may be secondary to improved mineral metabolism, by cinacalcet, that reduces the pro-calcification burden. This possibility was proposed in non-dialysed diabetic nephropathy patients [7].

Although we are aware of the main limitation of this preliminary study, the highly significant changes in both OPG and Fetuin-A levels observed in our patients provide a stimulus and starting point for further research in this field.

Acknowledgements. This study was supported by the grant: 'Project Glomerulonephritis' in memory of Pippo Neglia.

Conflict of interest statement. Dr Piergiorgio Messa received lecture fees from AMGEN, ABBOTT, and DOMPE' BIOTEC. The other authors have no conflict of interest to declare.

${ }^{1}$ Nephrology, Dialysis and

Transplant Unit, Department of Medicine and

${ }^{2}$ Clinical Pathology Laboratory Ospedale Maggiore-PoliclinicoMaRe-IRCCS,

Fondazione, Milano, Italy

Email: pmessa@policlinico.mi.it

1. Moe SM, Chertow GM, Coburn JW. Achieving NKF-K $\mathrm{DOQI}^{\mathrm{TM}}$ bone metabolism and treatment goals with cinacalcet HCl. Kidney Int 2005; 67: 760-771

2. Ketteler M. Fetuin-A and extraosseous calcification in uremia. Curr Opin Nephrol Hypertens 2005; 14: 337-342
3. Kiechl S, Schett G, Wenning G et al. Osteoprotegerin is a risk factor for progressive atherosclerosis and cardiovascular disease. Circulation 2004; 109: 2175-2180

4. Pritzker LB, Scatena M, Giachelli CM. The role of osteoprotegerin and tumor necrosis factor-related apoptosis-inducing ligand in human microvascular endothelial cell survival. Mol Biol Cell 2004; 15: 2834-2841

5. Morena M, Terrier N, Jaussent I et al. Plasma osteoprotegerin is associated with mortality in hemodialysis patients. $J$ Am Soc Nephrol 2006; 17: 262-270

6. Stenvinkel P, Wang K, Qureshi AR et al. Low Fetuin-A levels are associated with cardiovascular death: impact of variations in the gene encoding fetuin. Kidney Int 2005; 67: 2383-2392

7. Mehrotra R, Westenfeld R, Christenson P et al. Serum Fetuin-A in non-dialysed patients with diabetic nephropathy: relationship with coronary artery calcification. Kidney Int 2005; 67: $1070-1077$

doi:10.1093/ndt/gfm312

Advance Access publication 25 May 2007

\section{Does size matter?}

Sir,

In the November 2006 issue of JASN, Opelz et al. published their analysis of the association of ACEI/ARB with patient and graft survival after renal transplantation utilizing the CTS registry [1]. In contrast to our study, published in JASN in March 2006, Opelz and colleagues reported that they failed to find such an association [2].

How can that be?

(i) There are differences in the group definitions and inclusion criteria between the two studies. While we included all patients transplanted between 1990 and 2003 with a functioning graft 3 months after transplantation, Opelz et al. used only patients transplanted between 1995 and 2004, with functioning graft 1 year after transplantation.

(ii) There are differences in the way ACEI/ARB enters the analysis. While we used ACEI/ARB intake as a timedependent variable, and only for graphical illustration divided our patients into those who had received ACEI/ ARB treatment after transplantation and those who had never received such treatment, Opelz et al. used ACEI/ $\mathrm{ARB}$ in a fixed manner, comparing groups based on ACEI/ARB treatment at the time of 1 year after transplantation. In order to compare these results to ours, we performed a re-analysis of our database, including only patients transplanted from 1995 on and only those who had a functioning graft 1 year after transplantation. Then we used the same group definition as Opelz and colleagues and compared our new results to their and our published ones. We obtained the following survival curves (restricted to 6 years of follow-up, as in the publication of Opelz et al.) (Figure 1).

At 6 years of follow-up, the survival rates are comparable (Table 1).

We computed the crude (unadjusted for confounding) hazard ratio (HR) for ACEI/ARB use with the reduced data base, for graft and patient survival. These hazard ratio estimates compare to the results based on time-varying entry of ACEI/ARB use are displayed in Table 2. 